\title{
Concordance and survival implications of preoperative subclassification of T3 rectal cancers by depth of mesorectal invasion using a 5-mm cut-off point with endorectal ultrasound and magnetic resonance imaging
}

\author{
Stephanie García Botello ${ }^{1,2} \wedge$, Rosa Martí Fernández ${ }^{1 \wedge}$, Coral Cozar Lozano ${ }^{\wedge}$, Salvador Campos Salher ${ }^{3}$, \\ Jose Martín Arévalo ${ }^{1,2} \wedge$, David Moro Valdezate ${ }^{1,2} \wedge$, Vicente Pla Martii ${ }^{1,2} \wedge$, Alejandro Espí Macías ${ }^{1,2}$ \\ ${ }^{1}$ Colorectal Surgery Unit, Biomedical Research Institute INCLIVA, Universitary Clinic Hospital, Valencia, Spain; ${ }^{2}$ Department of Surgery, \\ University of Valencia, Valencia, Spain; ${ }^{3}$ Imaging Department, ERESA, Universitary Clinic Hospital, Valencia, Spain
}

Contributions: (I) Conception and design: S García Botello, J Martín Arévalo, D Moro Valdezate; (II) Administrative support: C Cozar Lozano, S Campos Salher, V Pla Martí, A Espí Macías; (III) Provision of study materials or patients: R Martí Fernández, S Campos Salher, V Pla Martí, A Espí Macías; (IV) Collection and assembly of data: S García Botello, R Martí Fernández, C Cozar Lozano, J Martín Arévalo; (V) Data analysis and interpretation: S García Botello, J Martín Arévalo, D Moro Valdezate; (VI) Manuscript writing: All authors; (VII) Final approval of manuscript: All authors.

Correspondence to: Rosa Martí Fernández, MD. Staff Colorectal Surgeon, Colorectal Surgery Unit, Department of General and Digestive Surgery, Universitary Clinic Hospital, Valencia, Spain. Email: rosamartifernandez@gmail.com.

Background: Validated rectal cancer staging groups T3 tumours in a single stage and depth of mesorectal invasion subclassification is not standard practice. Our aim is to report concordance between magnetic resonance imaging (MRI) and endorectal ultrasound (ERUS) for preoperative staging of T3 rectal tumours using a 5 -mm cut-off point and possible survival implications.

Methods: Prospective cohort study including patients staged preoperatively as cT3 by ERUS or magnetic resonance imaging. The maximum depth of penetration beyond the outer longitudinal muscle layer was measured according to a 5 -mm cut-off point. Concordance rate and Kappa coefficient were calculated for both techniques. Primary end-points were disease free survival and overall survival (OS) for both groups.

Results: A total of 97 patients were included. Disease-free survival in depth of mesorectal invasion $\leq 5$ and $>5 \mathrm{~mm}$ measured by ERUS was 130.80 (119.20-142.30) vs. 88.38 (56.13-120.64) months $(\mathrm{P}=0.020)$, respectively, and 129.90 (117.90-141.90) vs. 93.60 (64.50-122.70) months $(\mathrm{P}=0.045)$ when measured by magnetic resonance imaging. Depth of mesorectal invasion $\leq 5 \mathrm{~mm}$ measured by ERUS and MRI was a prognostic factor for both OS [ERUS P=0.009; MRI P=0.019] and DFS (ERUS P=0.026; MRI P=0.054) after Cox regression analysis.

Conclusions: T3 subclassification above and below $5 \mathrm{~mm}$ is feasible by ERUS, shows good concordance with validated magnetic resonance and can easily be incorporated into the diagnostic workup for these patients with possible survival implications.

Keywords: Depth of mesorectal invasion (DMI); rectal cancer; endorectal ultrasound (ERUS); magnetic resonance imaging (MRI); T3 tumours

\footnotetext{
^ ORCID: Stephanie García Botello, 0000-0001-6921-4902; Rosa Martí Fernández, 0000-0002-8061-5421; Coral Cozar Lozano, 00000002-2793-3164; Jose Martín Arévalo, 0000-0001-5337-9577; David Moro Valdezate, 0000-0003-0425-8677; Vicente Pla Martí, 00000001-7644-8416.
} 
Submitted Sep 03, 2021. Accepted for publication Dec 22, 2021; Published online: 17 Jan 2022.

doi: 10.21037/qims-21-880

View this article at: https://dx.doi.org/10.21037/qims-21-880

\section{Introduction}

The current rectal cancer staging systems such as the TNM Classification of Malignant Tumors (TNM) and American Joint Committee on Cancer (AJCC) based on tumour infiltration, lymph node invasion and metastatic spread, are currently the standard for colorectal cancer and reflect prognosis (1). Pathological T3 rectal cancers defined as depth of mesorectal invasion (DMI) beyond the muscularis propria are a heterogeneous group which are normally classified in a single-stage category. There have been several proposals for subclassifying them as T3a-T3d (2-4) but this has not become standard practice and is presently an optional rather than a compulsory reporting item (5). However, there is a need to substratify these tumours, finetune the indications for preoperative neoadjuvant therapy and avoid unnecessary pelvic radiation and long-term complications.

Several authors have reported the prognostic influence of postoperative histopathological DMI regardless of lymph node status and have suggested that this parameter should be included in therapeutic decision making $(6,7)$. Three-, 4 , 5 - and 6-mm cut-off points have been studied and proposed as independent risk factors for tumour recurrence and longterm survival (6-12). The 5-mm cut-off point proposed by Merkel et al. $(3,4)$ has recently gained more popularity and there are a handful of studies reporting the value of this histopathological cut-off point $(10,13)$ as an independent prognostic indicator.

Preoperative or clinical subclassification of T3 rectal tumours was assessed in the MERCURY trial and the magnetic resonance imaging (MRI) measurement of DMI has been shown to be precise and equivalent to histopathological measurements (14-16). The preoperative MRI based DMI of 4- and 5-mm cut-off point have been used $(13,15,17)$ with promising results. Endorectal ultrasound (ERUS) has long been regarded as a valuable staging method in local assessment of primary rectal cancer. ERUS has been proposed as a locoregional sating method by identifying tumours which may benefit from surgery alone $v s$. neoadjuvant therapy (18), moreover, it allows the identification of the circumferential resection margin for anterior tumours (19) or the subclassification of T3 tumours according to maximum tumour thickness (20) with high accuracy when compared to histopathological findings. However, ERUS subclassification of T3 tumours according to DMI has been scarcely reported $(15,21)$. The hypothesis of this study is that ERUS has a good concordance with MRI for preoperative staging of T3 rectal tumours and could be used as a prognostic factor.

We present the following article in accordance with the STROBE reporting checklist (available at https://qims. amegroups.com/article/view/10.21037/qims-21-880/rc).

\section{Objectives}

We aimed to investigate the concordance between MRI and ERUS for preoperative staging of T3 rectal tumours using the $5 \mathrm{~mm}$ cut-off point and its role as a prognostic indicator.

\section{Methods}

\section{Study design and population}

A prospective consecutive cohort single centre study was carried out in patients undergoing definitive curative surgery for rectal cancer from 2008 through 2018 and followed up until March 2020. Data was collected from a prospective institutional endorectal ultrasound database and subsequently analysed. Surgery was performed by one of 5 members of a highly specialized colorectal unit from a tertiary hospital, the Universitary Clinic Hospital of Valencia (Spain). The study was conducted in accordance with the Declaration of Helsinki (as revised in 2013). The study was approved by Institutional Review Board of Universitary Clinic Hospital and informed consent was taken from all individual participants. Patients with clinical T3 rectal tumours (less than $15 \mathrm{~cm}$ from the anal verge by rigid proctoscopy) were consecutively selected. We considered tumors of the lower third of the rectum up to $7 \mathrm{~cm}$ from the anal margin, of the middle third between 7.1 and $12 \mathrm{~cm}$, and of the upper third those located between 12.1 and $15 \mathrm{~cm}$.

All patients underwent pre-treatment staging by both ERUS and MRI less than 4 weeks before first treatment (either surgery or neoadjuvant therapy), and those with a T3 diagnosis by either technique were included 
regardless of lymph node status. Exclusion criteria were emergency surgery, more than one primary site, synchronous metastasis, peritoneal carcinomatosis at the time of surgery, a local transanal excision, and if they developed metachronous tumours. Patients in whom both techniques staged the tumour other than T3 or either of the radiological techniques could not be performed (patient or technical related factors) were also excluded from the study. Indications for neoadjuvant or adjuvant treatment are discussed at the weekly multidisciplinary team, and are individualized for each patient. General indications for treatment are based on patient performance status, tumour distance from the anal verge, presence of lymph nodes, perineural and vascular invasion, and circumferential resection margin involvement. The standard neoadjuvant chemotherapeutic regiments used were a total of 45-50 Gy in 25-28 fractions and concomitant capecitabine $825 \mathrm{mg} / \mathrm{m}^{2}$ oral every $12 \mathrm{~h}$, or short course of radiotherapy $25 \mathrm{~Gy}$ over 5 days ( $5 \mathrm{~Gy} /$ fraction) without preoperative chemotherapy. Adjuvant therapy regimen used was FOLFOX, a total of 6 postoperative cycles.

\section{Data collection and follow-up}

Patient follow-up was performed with 6 monthly serum carcinoembryonic antigen (CEA) levels, rigid proctoscopy and chest/abdominal computed tomography scan performed after elevation of CEA levels or at yearly intervals if the latter were normal. The outcome variables were: (I) concordance between MRI and ERUS measurements of DMI and (II) 5-year disease-free survival (DFS). Other variables analysed were age, sex, American Society of Anesthesiologists (ASA) score, tumour stage, lymph nodes, lymphovascular and perineural invasion, resection margins, overall survival (OS) and 1- and 2-year local recurrence.

ERUS was performed on an outpatient basis, rectal preparation was performed with a $250-\mathrm{mL}$ rectal enema 2 hours before the procedure. A $6-16 \mathrm{MHz}$ multifrequency $360^{\circ}$ transducer (B \& K Medical Systems Pro Focus $2202^{\circledR}$ scanner and B-K 2050 probe, Herlev, Denmark) was used and all ultrasounds were performed by 1 of 2 surgeons with over 15 years' experience in the technique. Real-time dynamic interpretation was performed in two-dimensional (2D) followed by $3 \mathrm{D}$, using $0.2-\mathrm{mm}$ slices throughout the length of the rectum and producing 300 sequential images that were automatically reconstructed as a cube, which could be saved for subsequent interpretation. This automated reconstruction of the images reduces human error as the ultrasound probe does not need to be moved throughout the examination and can be subsequently saved, allowing post examination analysis of the 3D-EAUS scan in coronal, sagittal or axial planes as deemed necessary.

MRI evaluation was performed and interpreted by a radiologist specialized in rectal MRI with a $1.5-\mathrm{T}$, magnetic resonance whole-body imager (signa Echo-Speed Plus with EXCITE; GE Medical Systems, Milwaukee, WI, USA) with a maximal gradient capacity of $33 \mathrm{mT} / \mathrm{m}$ and an 8 -element pelvic phased array coil. Axial, sagittal, coronal and oblique planes with $\mathrm{T} 2$-weighted sequences, with torsopa coil and rectal washings prior to the test, in a $1.5-\mathrm{T}$ GE machine were obtained. Axial oblique sequence is field of view (FOV) $22 \mathrm{~mm}$, matrix $256 \times 256$ with a slice thickness of $3 \mathrm{~mm}$. Both ERUS and MRI interpreters were blinders as to the results of the other examination.

The standard rectal MRI protocol in the evaluation of rectal cancer includes performing 2D fast spin-echo (FSE) T2-weighted sequences without fat suppression, using a small FOV and a section thickness less than $3 \mathrm{~mm}$ (highresolution protocol). Images in this sequence should be obtained in the (I) oblique axial plane (perpendicular to the tumor), as incorrect plane obliquity leads to blurring of the muscularis propria, which can cause incorrect $T$ staging; (II) sagittal plane, which is determined by the longitudinal tumor axis; and (III) oblique coronal plane (parallel to the anal canal), which is important to depict low rectal tumors and to better evaluate their relationship with the anal sphincter. These sequences have a proven high diagnostic accuracy, between $90 \%$ and $100 \%$, for the evaluation of tumor invasion into the mesorectal fascia (MRF) and adjacent organs and are recommended by the Magnetic Resonance Imaging and Rectal Cancer European Equivalence (MERCURY) group (14). FSE T2-weighted MRI with a large FOV without fat suppression obtained in the axial plane of the entire pelvis, from the aortic bifurcation to the sphincter, allows for evaluation of distant lymph node chains (e.g., inferior mesenteric, lateral, and inguinal). In the sagittal plane, from one side of the pelvic wall to the other, FSE T2-weighted MRI allows for localization of the primary tumor, enabling the measurement of its height and its relationship to the midline structures, such as the anal verge. Routine bowel preparation is not recommended.

For each patient, the maximum DMI beyond the outer longitudinal muscle layer was measured on the workstation for both ERUS and MRI images using electronic calipers. Measurement of the maximum extramural depth from outer muscle layer to outer edge of tumour was recorded in 

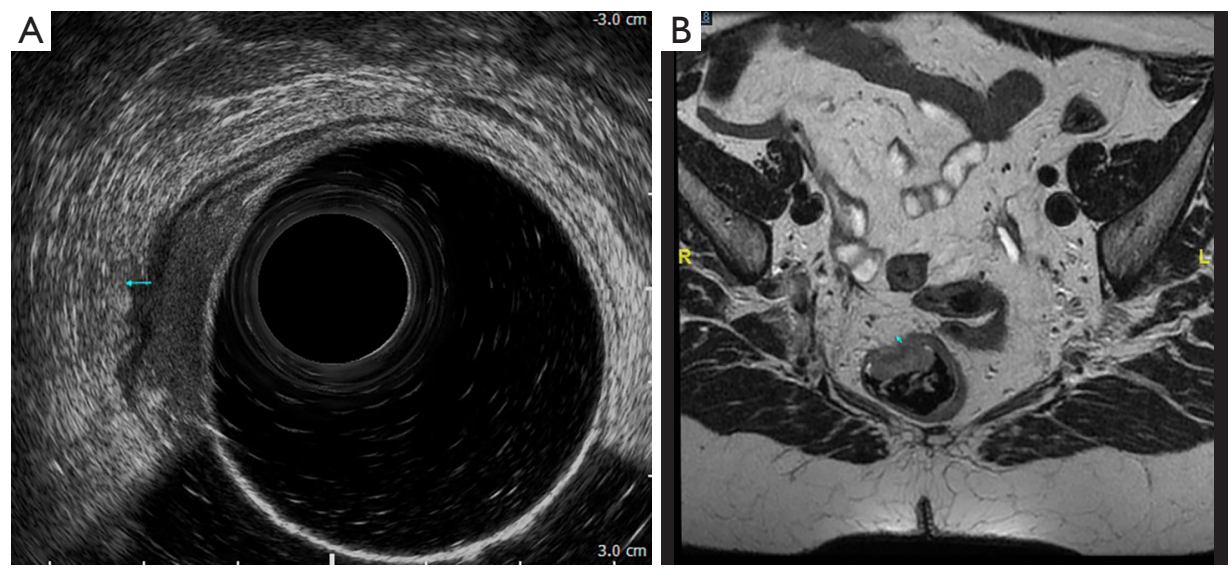

Figure 1 Early $[\leq 5 \mathrm{~mm}$ depth of mesorectal invasion (DMI)] T3 rectal tumour with a clearly visible muscularis propria. Arrow represents DMI. (A) Endorectal ultrasound. (B) Magnetic resonance imaging.
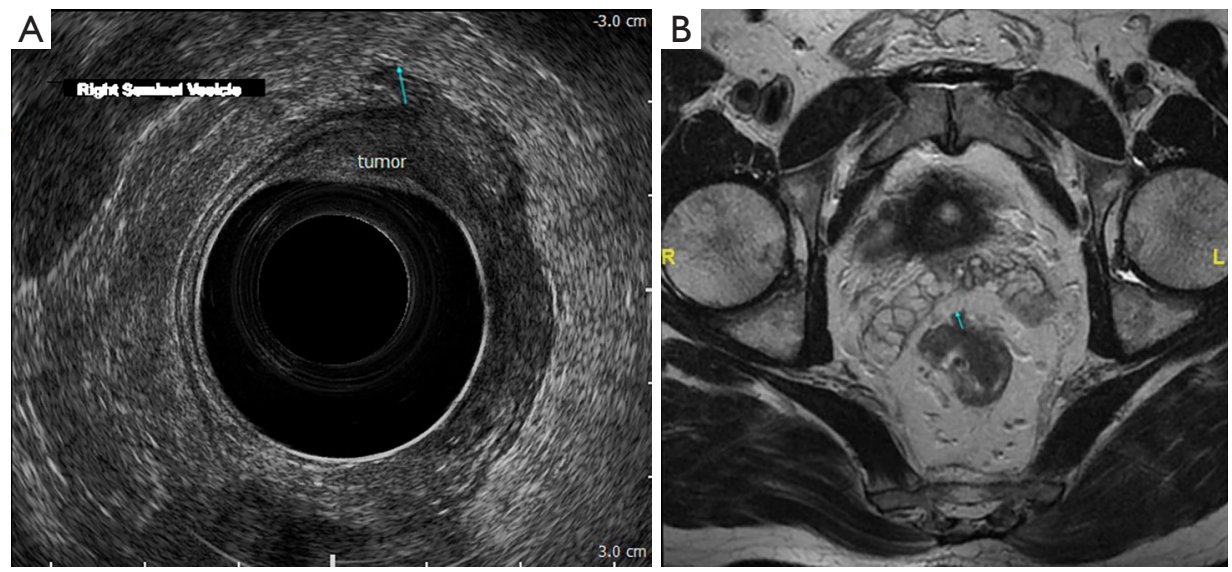

Figure 2 Early, nodular [ $\leq 5 \mathrm{~mm}$ depth of mesorectal invasion (DMI)] T3 rectal tumour with a partially interrupted muscularis propria. Arrow represents DMI. (A) Endorectal ultrasound imaging. (B) Magnetic resonance imaging.

millimetres. Maximum DMI was measured from the outer border of the muscularis propria (when identified) (Figures $1,2)$ to the deepest edge of mesorectal tumour invasion; if the muscularis propria could not be clearly identified due to destruction by tumour invasion or excessive inflammatory reaction, an imaginary line connecting both break points of the muscularis propria was traced, and the deepest edge of mesorectal tumour invasion was measured from this point (Figure 3) $(9,14,15)$. Patients were divided into DMI $\leq 5 \mathrm{~mm}$ and $\mathrm{DMI}>5 \mathrm{~mm}$.

\section{Statistical analysis}

A descriptive analysis was performed, and quantitative variables were tested for normality with the ShapiroWilk test. Qualitative data were expressed as n (\%) and quantitative data as median (range). The chi-squared test was used for categorical variables and U-Mann Whitney for comparison between continuous variables. The concordance rate and the Kappa coefficient between DMI measured by MRI and ERUS (degree of non-random agreement between different measurements of the same variable) were calculated. The Kappa coefficient varies between -1 and 1, considering: $\kappa=-1$, random match; $\kappa<0.2$, poor match; $\kappa=0.2-0.4$, low match; $\kappa=0.4-0.6$, moderate match; $\kappa=0.6-$ 0.8 , good match; $\kappa=0.8-1$, very good match. Pearson's correlation was used to demonstrate the relationship between measurements by both radiological techniques. 

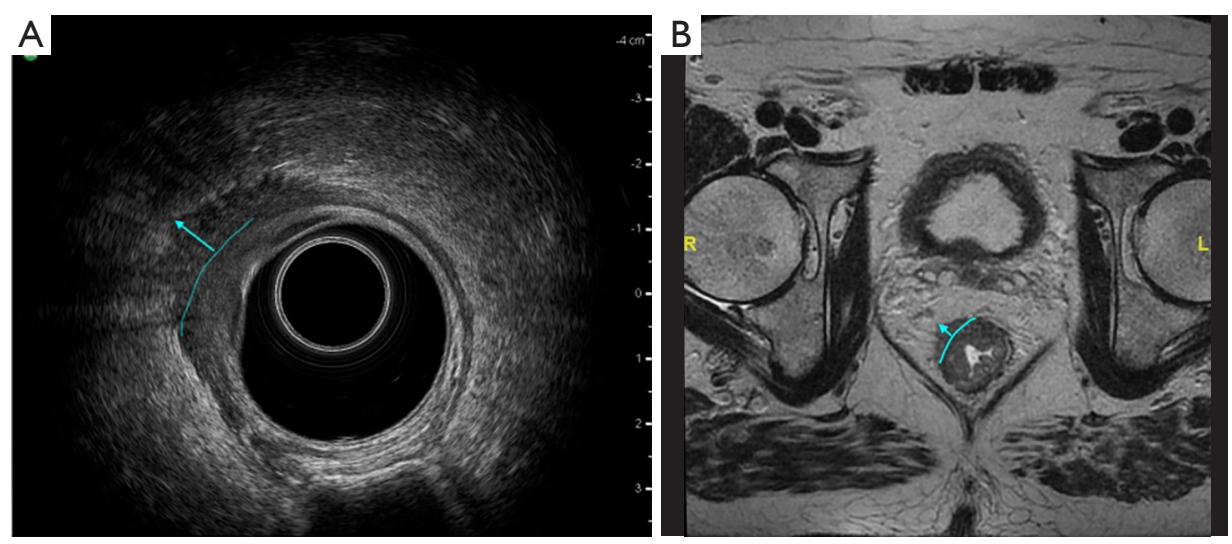

Figure 3 Advanced [ $>5 \mathrm{~mm}$ depth of mesorectal invasion (DMI)] T3 rectal tumour with an interrupted muscularis propria. Blue line represents imaginary muscularis propria, joining both breaking points. Arrow represents DMI. (A) Endorectal ultrasound imaging. (B) Magnetic resonance imaging.

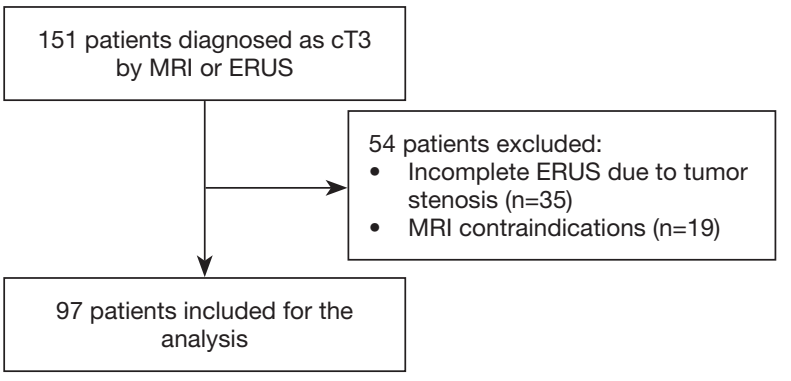

Figure 4 Study flow diagram. MRI, magnetic resonance imaging; ERUS, endorectal ultrasound.

The Bland-Altman method was used to compare DMI measured by ERUS and MRI.

DFS for either technique using the 5-mm DMI cut-off point was calculated using the Kaplan-Meier curves and log-rank test to assess for differences between curves. Cox regression analysis was used to assess if 5-mm DMI was prognostic for DFS and OS. Statistical analysis was carried with the $\mathrm{IBM}^{\circledR}$ SPSS $^{\circledast}$ Statistics Version 26 for MAC. $\mathrm{P}<0.05$ was considered statistically significant. Statistical tests were two-sided.

\section{Results}

A total of 151 patients staged as cT3 either by ERUS or MRI were initially included. After applying the exclusion criteria, a final cohort of 97 patients with a median age of 67.5 years (43-90 years), 64 (66.0\%) male was defined for the study (Figure 4). Median follow-up was 84 months. Forty- five (46.4\%) patients were classified as ASA score II, median distance of the tumour from the anal verge was $7.10 \mathrm{~cm}$ $(2-14 \mathrm{~cm}), 46(47.4 \%)$ were diagnosed as lower third rectal tumour, $45(46.4 \%)$ as middle third and $6(6.2 \%)$ as upper third (Table 1).

ERUS staged $6(6.2 \%)$ patients as T2, $89(91.8 \%)$ as T3 and $2(2.1 \%)$ patients as T4. MRI staged $2(2.1 \%)$ patients as T1, $9(9.3 \%)$ as $\mathrm{T} 2,84(86.6 \%)$ as $\mathrm{T} 3$ and $2(2.1 \%)$ as T4. Anal sphincter invasion was reported in $9(9.3 \%)$ by ERUS and in 4 patients (4.1\%) by MRI.

ERUS nodal status was N0 28 (28.9\%), N1 57 (58.8\%) and N2 12 (12.4\%), and by MRI: N0 24 (24.7\%), N1 48 (49.5\%) and N2 25 (25.8\%). Nineteen patients (19.6\%) developed a recurrence during the follow-up period, the majority of which were systemic 14 (14.4\%) patients. Thirty patients (30.9\%) died during the study period, where disease progression was the most common cause in 13 patients (13.4\%).

There was good concordance between ERUS and MRI when the DMI $\leq 5$-mm cut-off point was used with a kappa value of 0.775 , as shown in Table 2. Concordance was highest and "very good" for tumours located in the mid rectum, followed by "good" for lower rectal tumours and "moderate" for upper rectal tumours with kappa values of $0.872,0.698$ and 0.571 respectively (Table 2). The correlation between ERUS and MRI measurement of rectal wall invasion was $77.03 \%(\mathrm{P}<0.001$; 95\% CI: $0.6745042-$ 0.8406164). The Bland-Altman chart showed that most of the points were between the established agreement limits (Figure 5). The smallest differences in mean measurements between the two radiological techniques appeared in $\mathrm{T} 3$ 
Table 1 Patient and tumor characteristics according to imaging technique and depth of mesorectal invasion

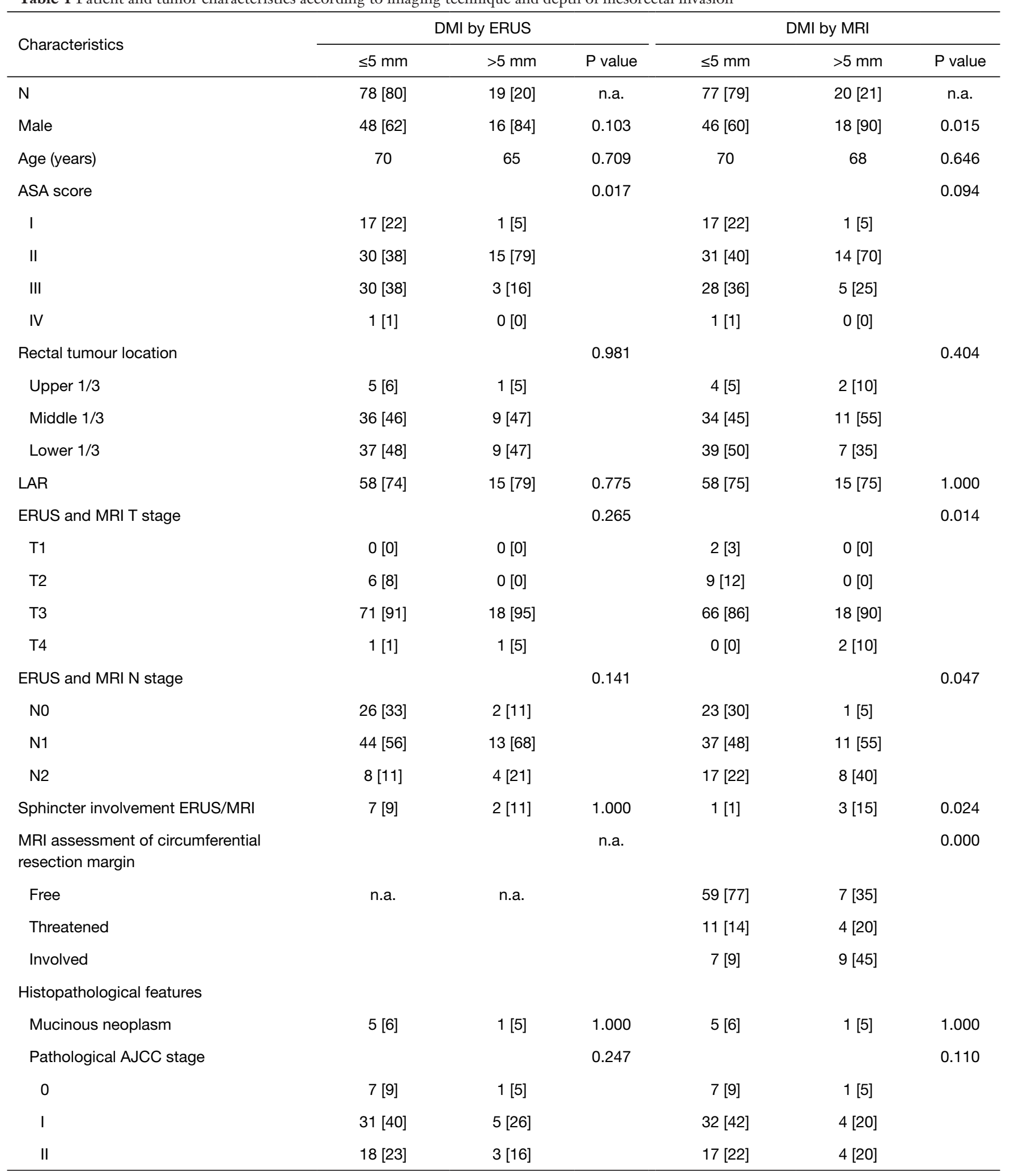

Table 1 (continued) 
Table 1 (continued)

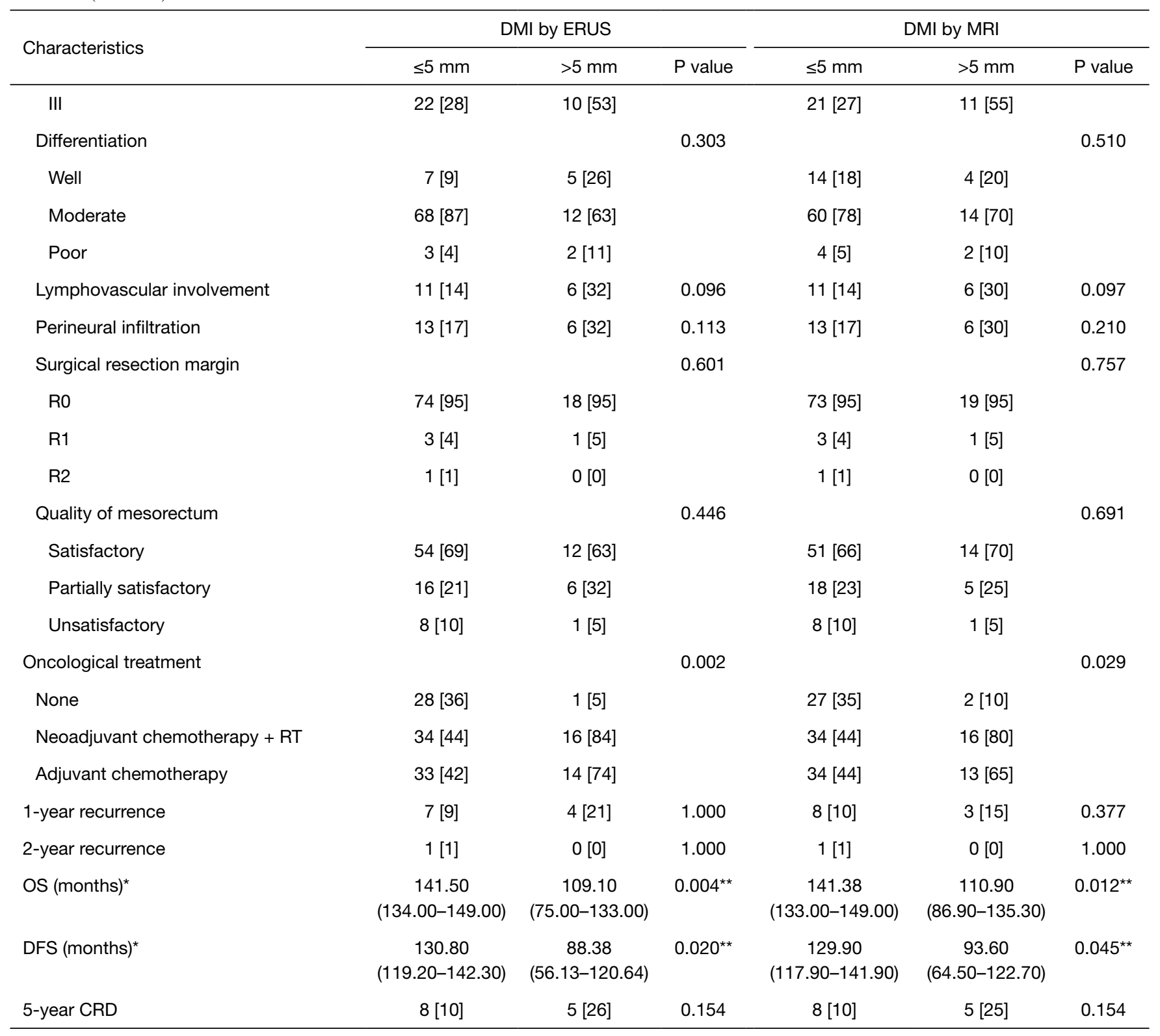

Results are expressed as $\mathrm{n}$ [\% DMI and imaging technique] unless otherwise stated. *, mean (95\% confidence intervals); ${ }^{* *}$, log rank analysis. OS, overall survival; DMI, depth of mesorectal invasion; DFS, disease-free survival; CRD, cancer-related death; MRI, magnetic resonance imaging; ERUS, endorectal ultrasound; AJCC, American Joint Committee on Cancer; n.a., not applicable; LAR, low anterior resection with primary anastomosis.

tumours with a parietal invasion less than or equal to $5 \mathrm{~mm}$. The mean difference in parietal invasion in $\mathrm{T} 3$ tumours was $0.3215 \mathrm{~mm}$ ( $95 \%$ CI: -0.293 to $0.936 \mathrm{~mm}$ ). This meant that, on average, ERUS measured $0.3215 \mathrm{~mm}$ more than MRI. The lower agreement limit was $-5.652 \mathrm{~mm}$ (95\% CI: -6.720 to $-4.590 \mathrm{~mm}$ ) and the upper agreement limit was
$6.300 \mathrm{~mm}$ (95\% CI: $5.232-7.360 \mathrm{~mm})$.

DFS and OS were calculated for the 5-mm DMI cut-off point. There were statistically significant differences with increased DFS and OS for the DMI $\leq 5 \mathrm{~mm}$ measured by ERUS and MRI, OS (ERUS $\mathrm{P}=0.004$; MRI $\mathrm{P}=0.012$ ) and DFS (ERUS P=0.020; MRI P=0.045) (Table 1 and Figure 6). 
Table 2 Kappa test results of concordance between MRI and ERUS for the whole group and stratifying according to location at the rectum

\begin{tabular}{|c|c|c|c|c|}
\hline Group & $\mathrm{MRI} \leq 5 \mathrm{~mm}$ & $\mathrm{MRI}>5 \mathrm{~mm}$ & Total & Kappa value \\
\hline \multicolumn{5}{|l|}{ Total group } \\
\hline ERUS $\leq 5 \mathrm{~mm}$ & 74 (76.3) & $4(4.1)$ & $78(80.4)$ & 0.775 \\
\hline ERUS $>5 \mathrm{~mm}$ & $3(3.1)$ & $16(16.5)$ & 19 (19.6) & \\
\hline Total & 77 (79.4) & $20(20.6)$ & 97 (100.0) & \\
\hline ERUS $\leq 5 \mathrm{~mm}$ & $4(66.7)$ & $1(16.6)$ & 5 (83.3) & 0.571 \\
\hline ERUS $>5 \mathrm{~mm}$ & $0(0)$ & 1 (16.7) & $1(16.7)$ & \\
\hline Total & $4(66.7)$ & 2 (33.3) & $6(100.0)$ & \\
\hline \multicolumn{5}{|l|}{ Middle third } \\
\hline Total & 34 (75.6) & $11(24.4)$ & $45(100.0)$ & \\
\hline \multicolumn{5}{|l|}{ Lower third } \\
\hline ERUS $\leq 5 \mathrm{~mm}$ & 36 (78.3) & $1(2.2)$ & $37(80.4)$ & 0.698 \\
\hline ERUS $>5 \mathrm{~mm}$ & $3(6.5)$ & $6(13.0)$ & 9 (19.6) & \\
\hline Total & 39 (84.8) & 7 (15.2) & $46(100.0)$ & \\
\hline
\end{tabular}

Values expressed as $\mathrm{n}$ (\% of total group). MRI, magnetic resonance imaging; ERUS, endorectal ultrasound.

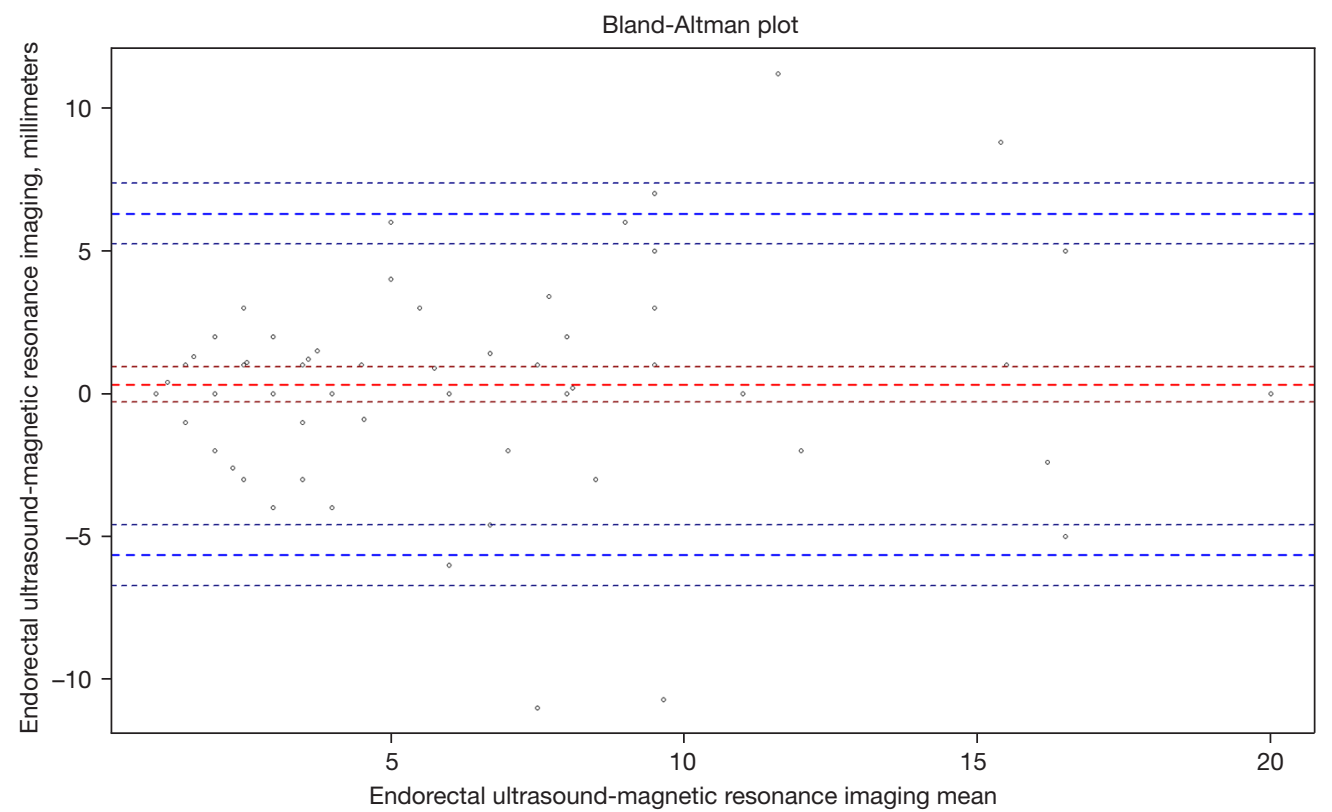

Figure 5 Bland-Altman chart comparing depth of mesorectal invasion measurement between endorectal ultrasound (ERUS) and magnetic resonance imaging (MRI). 

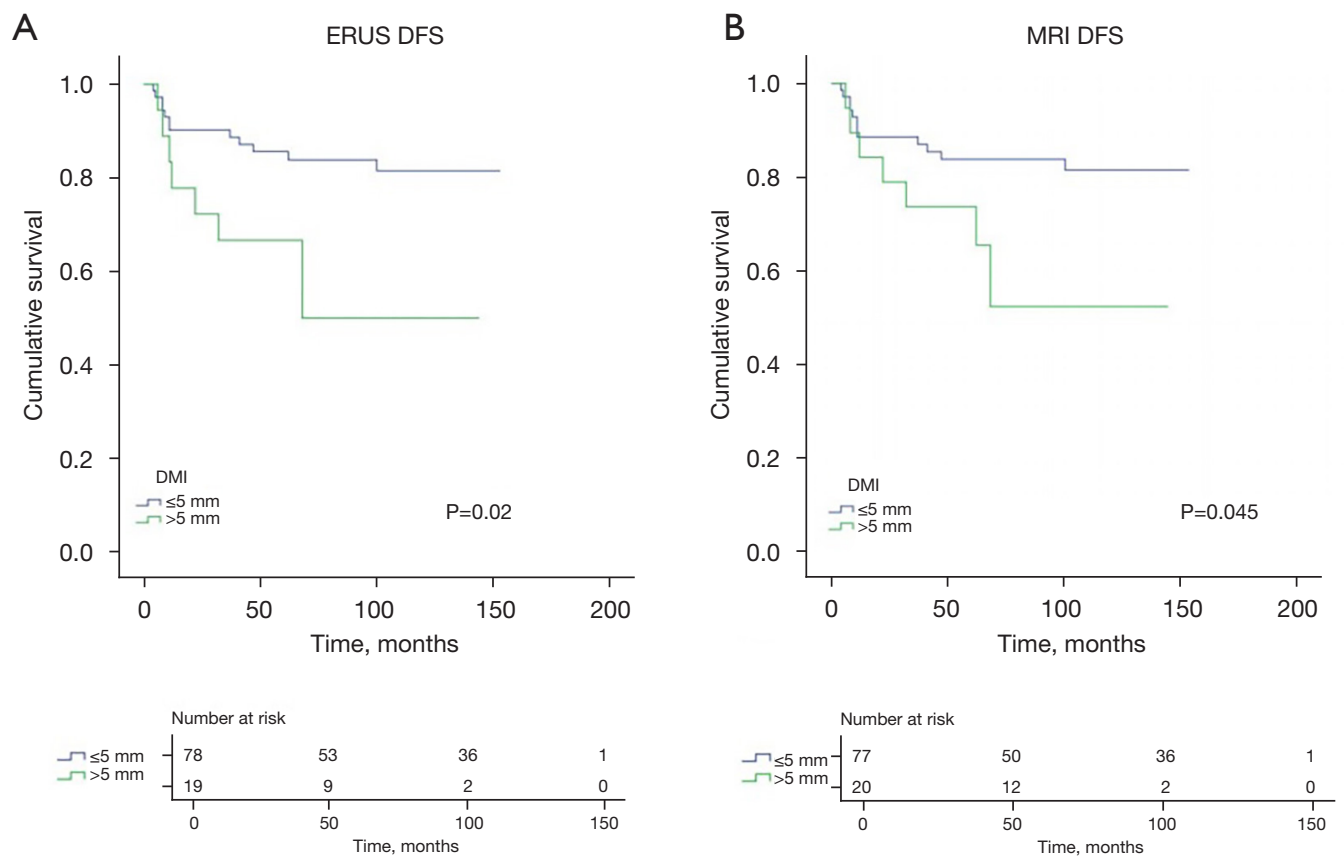

Figure 6 Kaplan-Meier curve showing disease-free survival (DFS) for 5-mm depth of mesorectal invasion (DMI). (A) Kaplan-Meier curve for DMI measured by endorectal ultrasound (ERUS). (B) Kaplan-Meier curve for DMI measured by magnetic resonance imaging (MRI).

Table 3 Cox regression analysis for T3 depth of mesorectal invasion $<5 \mathrm{~mm}$ by MRI and ERUS

\begin{tabular}{lccc}
\hline Variable & HR & $95 \% \mathrm{Cl}$ & P value \\
\hline OS & & & \\
MRI & 0.249 & $0.078-0.794$ & 0.019 \\
ERUS & 0.214 & $0.133-0.882$ & 0.009 \\
DFS & & & \\
MRI & 0.396 & $0.154-1.012$ & 0.054 \\
ERUS & 0.343 & $0.133-0.388$ & 0.026 \\
\hline
\end{tabular}

OS, overall survival; DFS, disease-free survival; MRI, magnetic resonance image; ERUS, endorectal ultrasound; DMI, depth of mesorectal invasion; $\mathrm{HR}$, hazard ratio; $\mathrm{Cl}$, confidence interval.

Univariant Cox regression analysis was performed for the variables OS and DFS, and DMI $\leq 5 \mathrm{~mm}$ measured by ERUS and MRI was found to be a prognostic factor (Table 3). Multivariant analysis was performed for the variables OS and DFS. DMI $>5 \mathrm{~mm}$ measured by ERUS was found to be independent prognostic factors for DFS $(\mathrm{P}=0.026)$ and for OS $(\mathrm{P}=0.013)$. DMI $>5 \mathrm{~mm}$ measured by MRI was not found to be an independent prognostic factor for DFS $(\mathrm{P}=0.054)$ or $\mathrm{OS}(\mathrm{P}=0.053)$.

\section{Discussion}

The present study shows that pre-treatment subdivision of T3 rectal tumours using a 5 -mm cut-off point offers a "good" overall concordance between ERUS and MRI measurements, which increases to a "very good" match for mid rectal tumours, "good" match for low rectal tumours and "moderate" for superior rectal cancers. The BlandAltmann plot showed an acceptable match between the ERUS and MRI measurement. MRI is currently the gold standard $(4,14,22-24)$ for pre-treatment staging of rectal cancer with reported accuracy ranging from $66 \%$ to $92 \%$ for $\mathrm{T}$ stage and from $60 \%$ to $90 \%$ for nodal metastases $(25,26)$, but these results show that the same measurements can be taken with ERUS and this latter technique could also be useful for clinically staging rectal tumours. ERUS has previously been shown to have an overall accuracy for $\mathrm{T}$ staging ranging from $62 \%$ to $92 \%$ and for lymph node metastases from $64 \%$ to $88 \%(18,20,21,27)$ and offers several advantages over MRI for pre-treatment cancer staging. It is widely available, and surgeon performed digital rectal exam and rigid proctoscopy are intrinsic components of the technique. The association of these three tools offers a very complete overview of tumour characteristics, distance from the anal verge and possibility of sphincter 
invasion. The higher accuracy rates reported for ERUS than for MRI in low rectal tumours $(19,22)$ could explain the lower concordance between ERUS and MRI for low $v s$. mid rectal tumours observed in this study. Conversely, ERUS has limited use in stenotic tumours, in the evaluation of the circumferential resection margin (19) and for assessing distant nodal invasion whereas MRI has a higher cost, longer exam duration and several contraindications such as patients with a pacemaker, intrauterine device, or claustrophobia (22). Our current policy is to use both ERUS and MRI as complementary techniques in the diagnostic work up of rectal tumours. To our knowledge, this is the first study comparing the concordance between MRI and ERUS for preoperative staging of $\mathrm{T} 3$ rectal tumours using a 5 -mm cut-off point and the findings assist in fine-tuning the indications for neoadjuvant therapy, avoiding unnecessary toxicity and improving overall oncological results.

The results of this study also serve to illustrate the prognostic importance of distinguishing between minimally invasive $(\leq 5 \mathrm{~mm})$ and advanced $(>5 \mathrm{~mm})$ T3 disease. There was a statistically significant increased DFS and OS in patients with DMI $\leq 5 \mathrm{~mm}$ when measured by both ERUS and MRI. These results are similar to those by Merkel et al., with cancer related 5 -year survival rate for pathological $\mathrm{DMI} \leq 5 \mathrm{~mm}$ and $\mathrm{DMI}>5 \mathrm{~mm}$ of $85 \%$ vs. $54 \%(\mathrm{P}<0.001)(3)$. ERUS measurement of DMI has been scarcely reported in the literature. Harewood et al. (21) described a 2-mm cutoff ERUS measurement of DMI for predicting survival and Zong et al. (15) reported a diagnostic accuracy of up to $86.9 \%$ for ERUS T3 sub-classification above or below $5 \mathrm{~mm}$ but did not use the results to predict survival. ERUS measurement of maximal tumour thickness (MTT) has also been used for substaging. Esclapez et al. (18) divided the T3 stage into uT3a (uMTT $\leq 19 \mathrm{~mm}$ ) and uT3b (uMTT $>19 \mathrm{~mm}$ ). We believe that this technique has some intrinsic problems in tumours with a large intraluminal component, which could be overstaged with this measurement and that ERUS of DMI is more reliable and comparable to MRI and pathological T3 substaging. Although DMI has been used to predict prognosis, there are few studies which report invasion of DMI measured by ERUS and its relation to survival $(15,21)$.

Patients with T3 rectal tumours represent a heterogeneous prognostic group. Five-year DFS ranges from $25-71 \%$, and DMI infiltration is one of the main prognostic factors (13). This is due to the higher probability of lymphovascular invasion and deposits of malignant cells in mesorectal fat in tumours with increased DMI (9). In
1993, the Union for International Cancer Control (UICC) proposed a cut-off value for DMI in pT3 rectal cancers in order to categorize pT3 subgroups and select the best treatment and follow-up for each patient (10). Several histopathological cut-off points have been chosen, such as $2 \mathrm{~mm}(21), 4 \mathrm{~mm}(6,9)$ or $6 \mathrm{~mm}(12)$. The 5 -mm cut-off point has been the most widely used and validated value for stratifying $\mathrm{T} 3$ patients $(3,13)$ both before and after neoadjuvant treatment (4). The 5-year recurrence rate for T3 rectal tumours in our study was $16.5 \%$, similar rates are reported in the literature ranging from $8.1 \%$ to $17.1 \%$, confirming the heterogeneity of T3 rectal tumour (28). After multivariant analysis, it seems that DMI $>5 \mathrm{~mm}$ measured by ERUS is an independent prognostic factor for OS and DFS.

There are several limitations to this study. It is a singlecentre study with MRI scans performed by one specialized radiologist and ERUS by two different surgeons. Interobserver variability was not assessed, though all scans and images were reviewed and assessed by all members of the multidisciplinary team during the weekly colorectal cancer meeting. Patients who had received neoadjuvant therapy were included in the study and therefore MRI and ERUS measurements were not compared to pathological assessment. These measurements have been widely validated in previous studies and we believe that this study simulates a more "real-life" scenario where initial treatment plans are based on clinical staging techniques and not on pathological specimen. Patients with DMI $>5 \mathrm{~mm}$ had a worse prognosis despite a larger number having received neoadjuvant therapy. The study required a wide time span in order to recruit a large enough cohort of patients with T3 tumours with both ERUS and MRI evaluation, as a proportion of patients were lost because either technique could not be performed due to technical difficulties. The strengths of this study are that it is the first study to report concordance rates between MRI and ERUS for T3 evaluation using a 5 -mm cut-off point and their respective significant survival implications. All imaging techniques were performed by experts in rectal MRI and ERUS and the relatively large number of patients and long rigorous follow-up are other significant strengths of the study.

\section{Conclusions}

In summary, T3 subclassification above and below $5 \mathrm{~mm}$ is feasible by ERUS, shows good concordance with validated MRI measurements and can easily be incorporated as a 
complementary technique into the diagnostic workup and reporting for these patients, with possible survival implications for rectal cancer patients.

\section{Acknowledgments}

Funding: None.

\section{Footnote}

Reporting Checklist: The authors have completed the STROBE reporting checklist. Available at https://qims. amegroups.com/article/view/10.21037/qims-21-880/rc

Conflicts of Interest: All authors have completed the ICMJE uniform disclosure form (available at https://qims. amegroups.com/article/view/10.21037/qims-21-880/coif). The authors have no conflicts of interest to declare.

Ethical Statement: The authors are accountable for all aspects of the work in ensuring that questions related to the accuracy or integrity of any part of the work are appropriately investigated and resolved. The study was conducted in accordance with the Declaration of Helsinki (as revised in 2013). The study was approved by Institutional Review Board of University Clinic Hospital and informed consent was taken from all individual participants.

Open Access Statement: This is an Open Access article distributed in accordance with the Creative Commons Attribution-NonCommercial-NoDerivs 4.0 International License (CC BY-NC-ND 4.0), which permits the noncommercial replication and distribution of the article with the strict proviso that no changes or edits are made and the original work is properly cited (including links to both the formal publication through the relevant DOI and the license). See: https://creativecommons.org/licenses/by-nc-nd/4.0/.

\section{References}

1. Edge SB, Greene FL, Byrd DR, Brookland RK, Washington MK, Compton CC. AJCC Cancer Staging Manual, 8th edition. Springer International Publishing: American Joint Commission on Cancer, 2016.

2. Cawthorn SJ, Parums DV, Gibbs NM, A'Hern RP, Caffarey SM, Broughton CI, Marks CG. Extent of mesorectal spread and involvement of lateral resection margin as prognostic factors after surgery for rectal cancer.
Lancet 1990;335:1055-9.

3. Merkel S, Mansmann U, Siassi M, Papadopoulos T, Hohenberger W, Hermanek P. The prognostic inhomogeneity in $\mathrm{pT} 3$ rectal carcinomas. Int J Colorectal Dis 2001;16:298-304.

4. Merkel S, Weber K, Schellerer V, Göhl J, Fietkau R, Agaimy A, Hohenberger W, Hermanek P. Prognostic subdivision of yp T3 rectal tumours according to extension beyond the muscularis propria. Br J Surg 2014;101:566-72.

5. Newland RC, Chapuis PH, Smyth EJ. The prognostic value of substaging colorectal carcinoma. A prospective study of 1117 cases with standardized pathology. Cancer 1987;60:852-7.

6. Shirouzu K, Akagi Y, Fujita S, Ueno H, Takii Y, Komori K, Ito M, Sugihara K; Japanese Society for Cancer of the Colon and Rectum (JSCCR) on Clinical Significance of the Mesorectal Extension of Rectal Cancer. Clinical significance of the mesorectal extension of rectal cancer: a Japanese multi-institutional study. Ann Surg 2011;253:704-10.

7. Yoshida K, Yoshimatsu K, Otani T, Yokomizo H, Ogawa K. The depth of tumor invasion beyond the outer border of the muscularis propria as a prognostic factor for T3 rectal/ rectosigmoid cancer. Anticancer Res 2008;28:1773-8.

8. Picon AI, Moore HG, Sternberg SS, Minsky BD, Paty PB, Blumberg D, Quan SH, Wong WD, Cohen AM, Guillem JG. Prognostic significance of depth of gross or microscopic perirectal fat invasion in T3 N0 M0 rectal cancers following sharp mesorectal excision and no adjuvant therapy. Int J Colorectal Dis 2003;18:487-92.

9. Akagi Y, Shirouzu K, Fujita S, Ueno H, Takii Y, Komori K, Ito M, Sugihara K; Study Group of the Japanese Society for Cancer of the Colon and Rectum (JSCCR) on the Clinical Significance of the Mesorectal Extension of Rectal Cancer. Predicting oncologic outcomes by stratifying mesorectal extension in patients with $\mathrm{pT} 3$ rectal cancer: a Japanese multi-institutional study. Int J Cancer 2012;131:1220-7.

10. Shin R, Jeong SY, Yoo HY, Park KJ, Heo SC, Kang GH, Kim WH, Park JG. Depth of mesorectal extension has prognostic significance in patients with $\mathrm{T} 3$ rectal cancer. Dis Colon Rectum 2012;55:1220-8.

11. Pollheimer MJ, Kornprat P, Pollheimer VS, Lindtner RA, Schlemmer A, Rehak P, Langner C. Clinical significance of $\mathrm{pT}$ sub-classification in surgical pathology of colorectal cancer. Int J Colorectal Dis 2010;25:187-96.

12. Miyoshi M, Ueno H, Hashiguchi Y, Mochizuki H, Talbot IC. Extent of mesorectal tumor invasion as a prognostic 
factor after curative surgery for $\mathrm{T} 3$ rectal cancer patients. Ann Surg 2006;243:492-8.

13. Siddiqui MRS, Simillis C, Bhoday J, Battersby NJ, Mok J, Rasheed S, Tekkis P, Abulafi AM, Brown G. A metaanalysis assessing the survival implications of subclassifying T3 rectal tumours. Eur J Cancer 2018;104:47-61.

14. MERCURY Study Group. Extramural depth of tumor invasion at thin-section MR in patients with rectal cancer: results of the MERCURY study. Radiology 2007;243:132-9.

15. Zhong G, Xiao Y, Zhou W, Pan W, Zhu Q, Zhang J, Jiang $Y$. Value of endorectal ultrasonography in measuring the extent of mesorectal invasion and substaging of T3 stage rectal cancer. Oncol Lett 2017;14:5657-63.

16. Cho SH, Kim SH, Bae JH, Jang YJ, Kim HJ, Lee D, Park JS; Society of North America (RSNA). Prognostic stratification by extramural depth of tumor invasion of primary rectal cancer based on the Radiological Society of North America proposal. AJR Am J Roentgenol 2014;202:1238-44.

17. Sueda T, Ohue M, Noura S, Shingai T, Nakanishi K, Yano M. Prognostic significance of a preoperative magnetic resonance imaging assessment of the distance of mesorectal extension in clinical T3 lower rectal cancer. Surg Today 2016;46:1249-57.

18. Esclapez P, Garcia-Granero E, Flor B, García-Botello S, Cervantes A, Navarro S, Lledó S. Prognostic heterogeneity of endosonographic T3 rectal cancer. Dis Colon Rectum 2009;52:685-91.

19. Granero-Castro P, Muñoz E, Frasson M, García-Granero A, Esclapez P, Campos S, Flor-Lorente B, Garcia-Granero E. Evaluation of mesorectal fascia in mid and low anterior rectal cancer using endorectal ultrasound is feasible and reliable: a comparison with MRI findings. Dis Colon Rectum 2014;57:709-14.

20. Muñoz E, Granero-Castro P, Frasson M, Escartin J, Esclapez P, Campos S, Flor-Lorente B, Garcia-Granero E. Modified Wong's classification improves the accuracy of rectal cancer staging by endorectal ultrasound and MRI. Dis Colon Rectum 2013;56:1332-8.

21. Harewood GC, Kumar KS, Clain JE, Levy MJ, Nelson H. Clinical implications of quantification of mesorectal tumor invasion by endoscopic ultrasound: All T3 rectal cancers are not equal. J Gastroenterol Hepatol 2004;19:750-5.

22. Kim YW, Cha SW, Pyo J, Kim NK, Min BS, Kim MJ,
Kim H. Factors related to preoperative assessment of the circumferential resection margin and the extent of mesorectal invasion by magnetic resonance imaging in rectal cancer: a prospective comparison study. World J Surg 2009;33:1952-60.

23. Taylor FG, Quirke P, Heald RJ, Moran B, Blomqvist L, Swift I, Sebag-Montefiore DJ, Tekkis P, Brown G; MERCURY study group. Preoperative high-resolution magnetic resonance imaging can identify good prognosis stage I, II, and III rectal cancer best managed by surgery alone: a prospective, multicenter, European study. Ann Surg 2011;253:711-9.

24. Beets-Tan RG, Beets GL, Vliegen RF, Kessels AG, Van Boven H, De Bruine A, von Meyenfeldt MF, Baeten CG, van Engelshoven JM. Accuracy of magnetic resonance imaging in prediction of tumour-free resection margin in rectal cancer surgery. Lancet 2001;357:497-504.

25. Brown G, Radcliffe AG, Newcombe RG, Dallimore NS, Bourne MW, Williams GT. Preoperative assessment of prognostic factors in rectal cancer using high-resolution magnetic resonance imaging. Br J Surg 2003;90:355-64.

26. Burton S, Brown G, Daniels I, Norman A, Swift I, Abulafi M, Wotherspoon A, Tait D. MRI identified prognostic features of tumors in distal sigmoid, rectosigmoid, and upper rectum: treatment with radiotherapy and chemotherapy. Int J Radiat Oncol Biol Phys 2006;65:445-51.

27. Garcia-Aguilar J, Pollack J, Lee SH, Hernandez de Anda E, Mellgren A, Wong WD, Finne CO, Rothenberger DA, Madoff RD. Accuracy of endorectal ultrasonography in preoperative staging of rectal tumors. Dis Colon Rectum 2002;45:10-5.

28. Bosset JF, Bosset M, Nguyen F, Servagi-Vernat S, Sedrati A. Defining preoperative treatment strategies in $\mathrm{t} 3$ rectal cancer. Gastrointest Cancer Res 2008;2:S54-7.

Cite this article as: García Botello S, Martí Fernández R, Cozar Lozano C, Campos Salher S, Martín Arévalo J, Moro Valdezate D, Pla Martí V, Espí Macías A. Concordance and survival implications of preoperative subclassification of T3 rectal cancers by depth of mesorectal invasion using a 5 -mm cut-off point with endorectal ultrasound and magnetic resonance imaging. Quant Imaging Med Surg 2022;12(4):23562367. doi: 10.21037/qims-21-880 